\title{
Interface Empresa-Universidade e a Gestão da Tecnologia
}

\author{
José Carlos Teixeira da Silva, Doutor em Engenharia (Escola Politécnica -USP) \\ Prof. Doutor, Depto. Eng. de Produção, Faculdade de Engenharia, Unesp, Campus de Bauru-SP \\ Av. Luiz Edmundo C. Coube S/N CEP 17033-000, Bauru-SP \\ jcteixe@bauru.unesp.br \\ José de Souza Rodrigues, Doutor em Engenharia (COPPE -UFRJ) \\ Prof. Doutor, Depto. Eng. de Produção, Faculdade de Engenharia, Unesp, Campus de Bauru-SP \\ Av. Luiz Edmundo C. Coube S/N CEP 17033-000, Bauru-SP \\ jsrod@bauru.unesp.br
}

\begin{abstract}
Este trabalho discute a interface empresa - universidade e a gestão da tecnologia em pequenas e médias empresas tradicionais do setor de manufaturados, com base em modelo conceitual simplificado e em pesquisa de campo, dentro do contexto atual de globalização do mercado. Constatou-se a importância da existência de um modelo de relação interorganizacional, que colabore na otimização dos resultados das pesquisas aplicadas na universidade e disponha de mecanismos de colaboração com aquelas empresas, visando à prática e difusão da gestão da tecnologia e parceria no desenvolvimento de produtos. Com base no modelo proposto em Silva(1999), através do COD - Centro Operacional de Desenvolvimento, o trabalho analisa vários aspectos da interface empresa - universidade e da gestão da tecnologia em empresas.
\end{abstract}

Palavras-chave: cooperação empresa-universidade; gestão da tecnologia; PMEs.

This paper discusses the interface enterprise-university and the manufacturing traditional small and medium enterprises. It attempts to analyze, through a simplified conceptual model and field research, the research in the university and the technology management in those industries, at the context of globalization. It was detected the importance to improve a inter-organizational model to optimize the researches in the university and the mechanisms of collaboration to those enterprises, with respect to the diffusion of practices in technology management and partnerships in integrated product development. It was proposed, based in Silva(1999), the DOCDevelopment Operational Center as an adequate management model to that industry-university interface.

Keywords: industry-university cooperation, technology management, SMEs.

\section{Introdução e Objetivos}

A equação do desenvolvimento, de acordo com Porter(1996), vem sendo modificada em seus parâmetros, pois dentre as quatro variáveis ligadas ao desenvolvimento, -terra, -capital, -trabalho e-tecnologia, apenas a tecnologia permanece ligada à competitividade. Segundo Gazzinelli(1999), em 1978 "a prioridade das empresas japonesas era aumentar a participação no mercado (35\%), reduzir custos (28\%) e desenvolver novos produtos (22\%). Hoje, a prioridade já se inverteu, pois $48 \%$ buscam desenvolver novos produtos e $34 \%$ aumentar a participação no mercado". Essa reviravolta no foco não está sendo acompanhada, ou pressentida, pelas empresas brasileiras, conforme recente estudo da CNI Confederação Nacional da Indústria, onde a preocupação das empresas ainda está voltada $80 \%$ para a capacitação de recursos humanos, $75 \%$ para a implantação de sistemas de qualidade e somente $25 \%$ para a capacitação da gestão tecnológica. Em termos de produção, $90 \%$ das empresas brasileiras estão voltando-se para a aquisição de máquinas e equipamentos visando o aumento da competitividade $e$ somente $15 \%$ disseram estar preocupadas com a de tecnologia. "Alguma coisa está errada no País, comenta Gazzinelli(1999), se apenas $25 \%$ das empresas acham que a capacitação tecnológica é o mais importante. Isso tem que mudar. Capacitar recursos humanos é importante mas é preciso haver preocupação com a gestão tecnológica. É diferente 'ser' e 'estar' competitivo".

No contexto atual da economia globalizada são vários os aspectos de competitividade, mas a tecnologia de produto/processo está entre as dimensões de primeira ordem, independentemente do tamanho da empresa. Embora as grandes empresas disponham de maior estrutura e informação para o gerenciar esse campo, as novas tecnologias nem sempre são geradas nessas organizações. Por outro lado, as pequenas e médias empresas também devem estar conscientizadas da importância da gestão da tecnologia e disporem de 
mecanismos que as auxiliem nesse campo, como fator de sobrevivência no mercado.

$O$ presente trabalho analisa aspectos da interação empresauniversidade correlacionados à gestão da tecnologia em pequenas e médias empresas, através de alguns conceitos e pesquisa de campo em empresas e universidades, utilizando o modelo proposto em Silva(1999).

\section{Revisão Bibliográfica}

$A$ interface empresa-universidade vem sendo pesquisada com maior intensidade nas últimas duas décadas, visando aplicabilidade dos conhecimentos gerados na comunidade. Segundo Zagottis (1996), "os contratos de pesquisa e desenvolvimento são importantes nesse contexto", onde, de acordo com Silva(1999) "essa interface tem perspectivas importantes na gestão da tecnologia de produtos/processos e na geração de novos produtos e processos, sobretudo no setor de manufaturados em pequenas e médias empresas".

Em sua análise da universidade européia, projetada para as universidades brasileiras, Durham (1990) indica alteração das demandas com "a diminuição dos recursos orçamentários para as universidades, o estímulo às relações com o setor produtivo e a ampliação de processos de avaliação". Apesar dos esforços nessa direção, a complexidade dessa interface ainda prevalece nos dias atuais, pelas dificuldades nas relações interorganizacionais. A maioria das pesquisas aplicadas, nas universidades, de acordo com Silva(1996), é desenvolvida sem a participação de empresas, caracterizando-se mais como "atividade fim" do que como "atividade meio". Segundo Meneghel; Gomes (1996), os projetos com recursos públicos desenvolvidos pela Unicamp-Universidade Estadual de Campinas-SP no período 1982 a 1995, "ainda representavam 92,7 \% do total investido naquele período". Nos Estados Unidos, a situação é similar, embora em outra ordem de grandeza. Apesar dos esforços da National Science Fundation NSF, os problemas das relações interorganizacionais ainda persistem, além de aspectos éticos, de legislação, de direitos autorais, de gestão etc., como assinalam Bowers (1991) e Streharsky (1993). Na realidade, como assinalou Silva(1999), "a maioria dos investimentos em projetos de pesquisa aplicada, conduzidos em universidades, muitas vezes bem sucedidos internamente, não têm se materializado em benefício do sistema produtivo e da comunidade, quer seja no Brasil ou Exterior".
A otimização dos investimentos em P\&D tem sido um paradigma tanto para as universidades como para as empresas. A competitividade da indústria japonesa a partir dos anos 70, e mais recentemente de outros países asiáticos, a dissolução do bloco soviético, e outros aspectos, contribuiu para aquilo que se convencionou chamar de "globalização", abrindo o mercado para as grandes empresas dos países centrais. As empresas que se adaptaram a essa nova configuração, cognominadas de "empreendimento ou empresas de classe mundial", segundo a classificação de Merli (1994), expandiram a gestão da qualidade com a abordagem cliente-

fornecedor, no contexto de "comakership". Na década de 90, como abordam Silva; Plonski (1999), "com o nivelamento dessas empresas em aspectos de qualidade e produtividade, começa a despontar outra dimensão de competitividade relacionada à gestão da tecnologia”. As conseqüencias dessa conjuntura se refletem nas pequenas e médias empresas de todos os países, onde essa nova forma de gestão tem se tornado um verdadeiro paradigma. Segundo Kruglianskas (1996), em sua análise de pequenas e médias empresas, "por detrás dessas dimensões estão as tecnologias de gestão da empresa, interna e externamente, e as tecnologias dos produtos e processos". Com a dimensão tecnologia se agregando como fator de competitividade, a universidade tem a oportunidade de desempenhar parceria importante junto ao sistema produtivo, principalmente em países em desenvolvimento. Por outro lado, como relatam Moraes; Stal (1994) o meio universitário deve compreender que "a capacitação tecnológica e a inovação tecnológica são fundamentais para a competitividade, e que as empresas são os principais agentes de $P \mathcal{E} D$, em função de que sua motivação primordial é o lucro".

$\mathrm{Na}$ interação empresa - universidade a dimensão informação é relevante, envolvendo dados de mercado, empresas, tecnologias, produtos, recursos humanos e materiais, fornecedores, consumidores, agências de financiamento, além dos resultados das pesquisas na universidade. Todavia, a informação tecnológica não é facilmente acessada nesses sistemas de informação, pois exige decodificação. Um trabalho que vem sendo conduzido com relativo sucesso nesse campo é aquele desenvolvido no Institute for Innovation and Transfer da University Karlsruhe, Alemanha, fazendo uso de rede ("network") interdisciplinar através do "Software Laboratory Karlsruhe", de acordo com Link(1997). 
O modelo de pesquisa praticado nas universidades, no campo da tecnologia aplicada, pode ser caracterizado como "linear" ou "não linear". No modelo linear o enfoque das pesquisas não visa aplicação externa imediata, mas somente ao término da pesquisa. No modelo não linear as pesquisas são desenvolvidas em parceria visando aplicação externa simultânea. Assim, o modelo de pesquisa praticado está diretamente relacionado ao modelo de interação da universidade com a comunidade. Publicações recentes, referentes à interface universidadeindústria, na América do Norte, Europa, Austrália e Brasil, indicam alguma convergência para modelos de implementação, embora as dificuldades e complexidades ainda persistam. Assim, temos o modelo cíclico de Ziman (1991) relacionando ciência e tecnologia, o modelo "empreendimento" proposto por Grigg (1994), o modelo em forma de espiral circular de Schuetze (1996), o modelo baseado no chamado Triângulo de Sábato proposto por Sábato; Botana (1968) apud Plonski (1995), o modelo "Triple Helix of University-Industry-

Government Relations" de Etzkowitz; Leydesdorff(1998), e o modelo do "Centro Operacional de Desenvolvimento - COD" sugerido por Silva(1999) na interface empresauniversidade visando o desenvolvimento de novos produtos, entre outros. É interessante ressaltar que a partir de 1973 a National Science Foundation - NSF vem incentivando as universidades públicas americanas a criarem centros de pesquisa em parceria com indústrias, como os IUCRCs (Industry-University - Cooperative Research Centers) e os ERCs (Engineering Research Centers). Em sua pesquisa apoiada pela NSF, quando analisou 51 IUCRCs e 8 ERCs, Geisler (1995) concluiu que "a sustentabilidade da cooperação indústriauniversidade-governo depende essencialmente da arquitetura dos centros, ou seja, da estrutura e do processo, manejada ao longo do tempo, pelo relacionamento entre o tipo de cooperação e o nível de complexidade organizacional necessária".

\section{Conceituação e Metodologia}

O conceito de "tecnologia" tem sido expandido para várias áreas do conhecimento, com abordagens muitas vezes distintas. No presente trabalho vamos considerar o conceito tradicional, envolvendo as tecnologias de produto/processo envolvidas na transformação da matéria-prima em produto final. Nesse contexto também existem particularidades para cada produto ou processo em função do conteúdo tecnológico e aspectos de mercado/consumidor: A gestão dessas tecnologias envolve toda a organização e tem implicações internas e externas à empresa.

O presente trabalho utiliza conceitos desenvolvidos $\mathrm{em}$ pesquisa anteriormente realizada, referenciada em Silva(1999), onde foi concebido o Centro Operacional de Desenvolvimento - COD como modelo interativo empresauniversidade no desenvolvimento de produtos, envolvendo pequenas e médias empresas do setor de manufaturados. Considerando as dimensões Universidade, Indústria e Centro Operacional de Desenvolvimento-COD, podemos estabelecer um modelo conceitual simplificado de acordo com a Fig. 1, com as dimensões universidade e industria, além da relação interorganizacional simbolizada pelo COD.

As dimensões universidade e industria podem ser desdobradas em vários aspectos relacionados nas Figuras 2 e 3 que seguem. Por outro lado, em relação ao COD, sua característica principal é de ser um órgão híbrido, ou seja, não tem vínculo funcional ou administrativo com a universidade ou empresas, e não se caracteriza como um centro de pesquisa cooperativa, como acontece nos IUCRCs ou ERCs dentro das universidades públicas americanas. O COD não executa ou gerencia pesquisas, mas é um gerador de projetos parceria de pesquisa entre universidade e empresas. Sua função principal é o desenvolvimento de novos produtos para empresas, com tecnologias existentes ou novas tecnologias, cujo maior detalhamento pode ser acessado em Silva(1999).

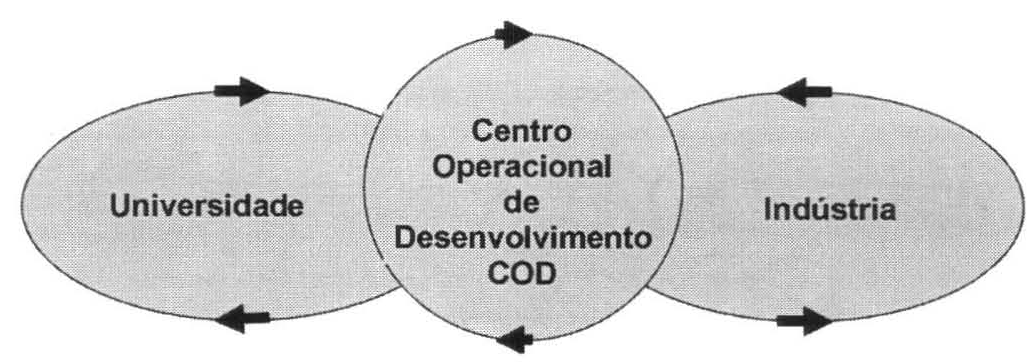

Figura 1. Dimensōes da Interface Empresa-Universidade 
A dimensão industria da Figura 2 incorpora aspectos da gestão da tecnologia em empresas e de interação empresa universidade, dentro do campo de tecnologia aplicada, ou seja, envolvendo principalmente as áreas de engenharia das universidades, sempre sob o ponto de vista das empresas tradicionais pesquisadas no setor de manufaturados.

A dimensão universidade da Figura 3 que segue incorpora aspectos das atividades de pesquisa da universidade, além das atividades de ensino relacionadas, e atividades de extensão. Nessa dimensão foram incluídos também os órgãos de cooperação universidade - empresa das universidades.

Tanto no que se refere à dimensão indústria, como a dimensão universidade, durante a análise dos resultados da pesquisa de campo, procuraremos destacar os aspectos mais relevantes dessas dimensões.

\section{DIMENSÃO INDÚSTRIA}

\begin{tabular}{|c|}
\hline Aspectos da Gestão da Tecnologia \\
\hline Planejamento estratégico de tecnologia na empresa \\
\hline Planejamento estratégico de produto / mercado na empresa \\
\hline Importância atual no mercado da empresa das tecnologias de produto e processo \\
\hline Relevância atual do desenvolvimento de produtos para a empresa \\
\hline Envolvimento contínuo ou temporário da empresa com pesquisa edesenvolvimento \\
\hline Origens das tecnologias de produto / processo utilizadas pela empresa \\
\hline Origem das idéias para desenvolvimento de novos produtos \\
\hline Estrutura organizacional dos grupos de desenvolvimento de produtos (se existentes) \\
\hline Participação das áreas funcionais no desenvolvimento de produtos \\
\hline Áreas funcionais que têm mais participação nas melhorias incrementais em produtos e processos \\
\hline Principais dificuldades para o desenvolvimento de novos produtos \\
\hline Etapas de maior importância atual no desenvolvimento de novos produtos para as empresas \\
\hline Participação no faturamento (últimos 5 anos) de novos produtos/processos/ melhorias incrementais \\
\hline Aspectos da Interação Empresa - Universidade \\
\hline Atividade de pessoal de nível superior compatível com a formação \\
\hline Adequação de formação de pessoal de nível superior para a empresa e treinamento \\
\hline Existência de técnico de nível médio no quadro de funcionários da empresa \\
\hline Principais áreas de interesse da empresa em cursos de especialização \\
\hline Participação da empresa em projetos parceria com a universidade \\
\hline Grau de satisfação da empresa quando realizou projeto parceria com a universidade \\
\hline Grau de recepção da universidade quando contatada pela empresa \\
\hline Interesse da empresa em desenvolvimento de produtos com a parceria da universidade via COD \\
\hline Disponibilidade na empresa de acesso a algum sistema de informação \\
\hline Conhecimento da empresa dos incentivos fiscais para pesquisa e desenvolvimento \\
\hline
\end{tabular}

Figura 2 - Aspectos da Dimensão Indústria 


\section{DIMENSÃO UNIVERSIDADE}

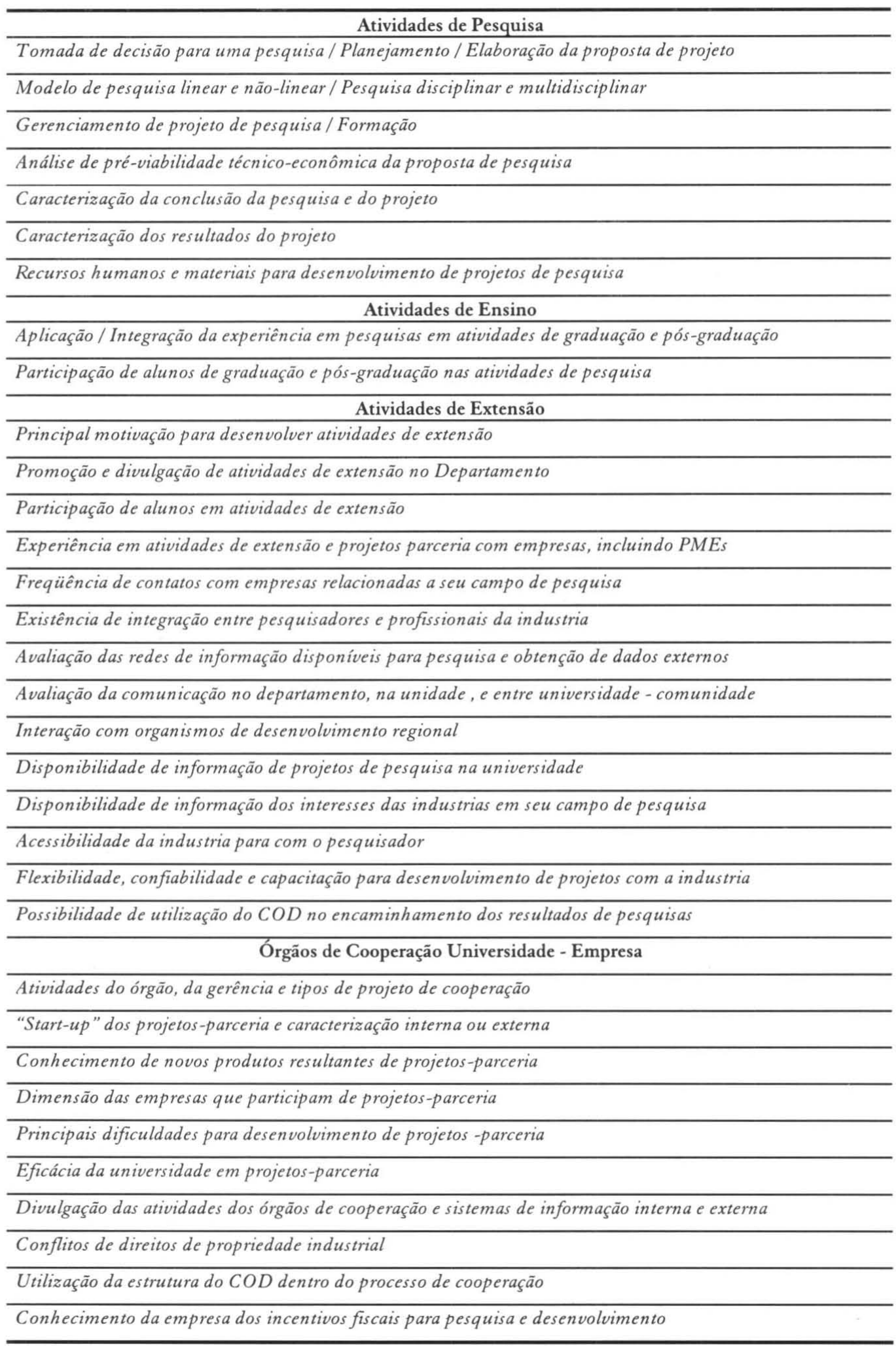

Figura 3- Aspectos da Dimensão Universidade 
Um esquema geral da gestão da tecnologia em empresas é apresentado na Fig. 4, onde temos a interface empresa-universidade envolvendo parceria $\mathrm{em}$ pesquisa, otimização e desenvolvimento de novos produtos/processos. O COD também desenvolve outras funções correlacionadas, como a difusão de informações, o treinamento de pesquisadores e profissionais de empresas em gerenciamento de projetos de pesquisal desenvolvimento, visando difundir a prática moderna de desenvolvimento de produtos. Em função da disponibilidade ou não das tecnologias de produto e de processo, a empresa deve estabelecer políticas e se envolver com uma série de procedimentos para ambas as alternativas. No caso de tecnologias disponíveis, sua reavaliação com relação a aspectos de competitividade de mercado, de meio ambiente, conservação de energia etc. Quando da não disponibilidade das tecnologias para um novo produto elou processo se inicia os procedimentos de prospecção elou desenvolvimento. Essa fase poderá contar com apoio do COD no caso de desenvolvimento $e$ da universidade quando envolver projetos parceria de pesquisa dentro do modelo não linear, culminando com o desenvolvimento, fabricação e lançamento do novo produto.

As opçōes de adquirir ou desenvolver tecnologias competitivas para produtos e processos definem a política da empresa no campo da gestão da tecnologia. Um aspecto importante nesse campo, visando minimizar riscos, é a análise da vulnerabilidade tecnológica para produtos e processos, face à concorrência, envolvendo situações como as indicadas na Figura 5 que segue, e adotando retaguardas diversas, entre elas o registro de propriedade industrial. O termo vulnerabilidade "baixa" é temporal e praticamente inexistente.

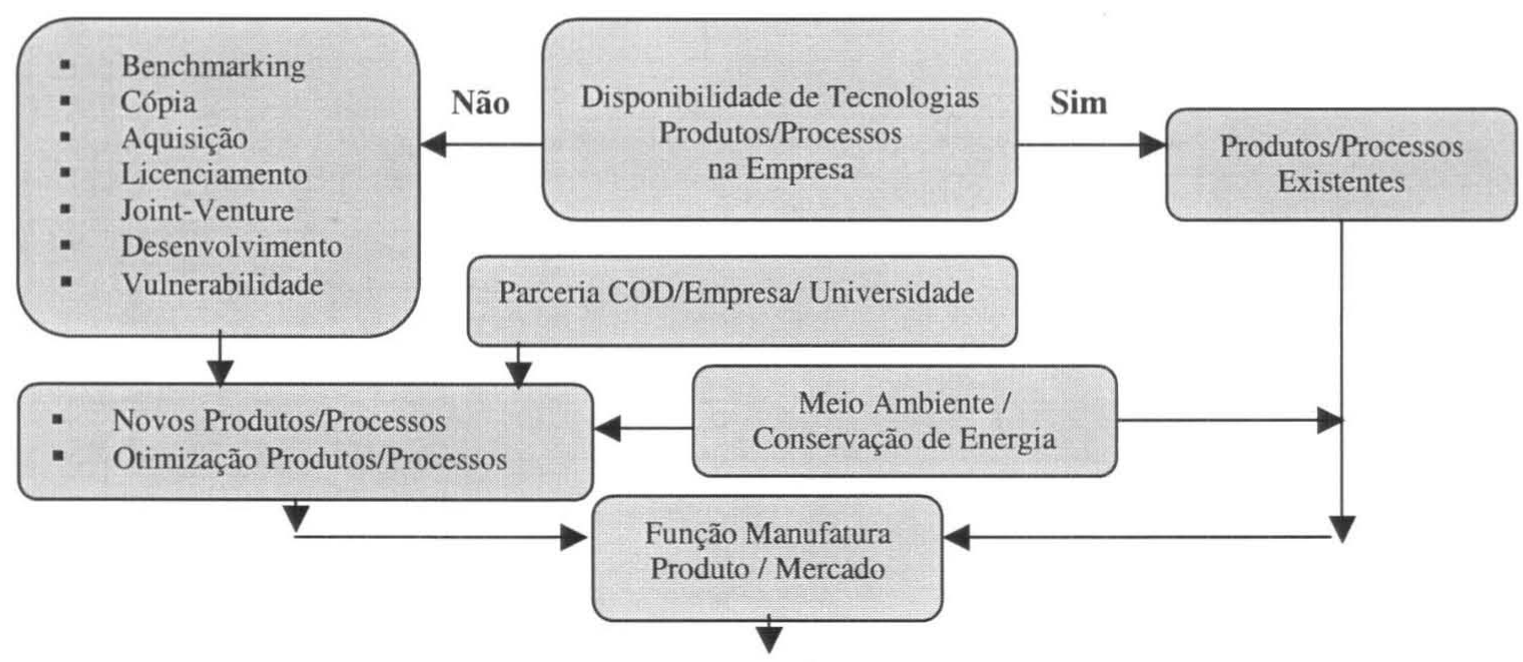

Figura 4 - Aspectos da Gestão da Tecnologia na Interface Empresa - Universidade

\begin{tabular}{c|c|c|c}
\hline & Produto & Processo & Vulnerabilidade \\
\hline $\begin{array}{c}\text { Tecnologia } \\
\text { Conhecida }\end{array}$ & $\begin{array}{c}\text { Domínio Público } \\
\text { Domínio Restrito } \\
\text { Domínio Restrito }\end{array}$ & $\begin{array}{c}\text { Domínio Público } \\
\text { Domínio Público } \\
\text { Domínio Restrito }\end{array}$ & $\begin{array}{c}\text { Alta } \\
\text { Mádia } \\
\text { Baixa }\end{array}$ \\
\hline $\begin{array}{c}\text { Nova } \\
\text { Tecnologia }\end{array}$ & Domínio Restrito & Domínio Público & Média \\
& Domínio Restrito & Baixa \\
\hline
\end{tabular}

Figura 5 - Vulnerabilidade da Tecnologia na Empresa (Silva; Plonski -1999) 
A conceituação desenvolvida permitiu análise baseada em referências bibliográficas e pesquisa de campo, através de metodologia com abordagem qualitativa no campo da pesquisa organizacional, envolvendo inicialmente o projeto de pesquisa "levantamento survey" e o método de pesquisa "questionário autoadministrado", dentro da conceituação de Bryman (1989). Após a análise do "survey", foi conduzido o projeto de "pesquisa qualitativa" em empresas $e$ universidades, através do método de pesquisa "entrevistas semi-estruturadas". A amostragem para o "survey" foi intencional, de caráter regional, envolvendo 50 pequenas empresas (20 a 100 funcionários) e 40 médias empresas (100 a 500 funcionários), associadas ao CIESP-Centro das Indústrias do Estado de São Paulo em 27 cidades, incluindo a capital. As pesquisas nas universidades foram conduzidas na USP-Universidade de São Paulo, UNICAMP-Universidade de Campinas, $e$
UNESP-Universidade Estadual Paulista. O "survey" utilizou escalas nominais e ordinais tipo Likert(1935) apud Hayes(1992), seguindo na análise a orientação de Siegel(1975). Os dados da pesquisa, incluindo a relação das empresas, bem como os métodos de análise dentro da área da estatística não-paramétrica poderão ser consultados em Silva(1999).

\section{Análise dos Resultados da Pesquisa de Campo}

A análise da pesquisa de campo "survey" envolve aspectos da gestão da tecnologia nas empresas e da interação empresa - universidade. Inicialmente, as Figuras 6 a 10 analisam o comportamento em aspectos da gestäo da tecnologia, apresentando predominância $e$ mediana, em função da escala utilizada.

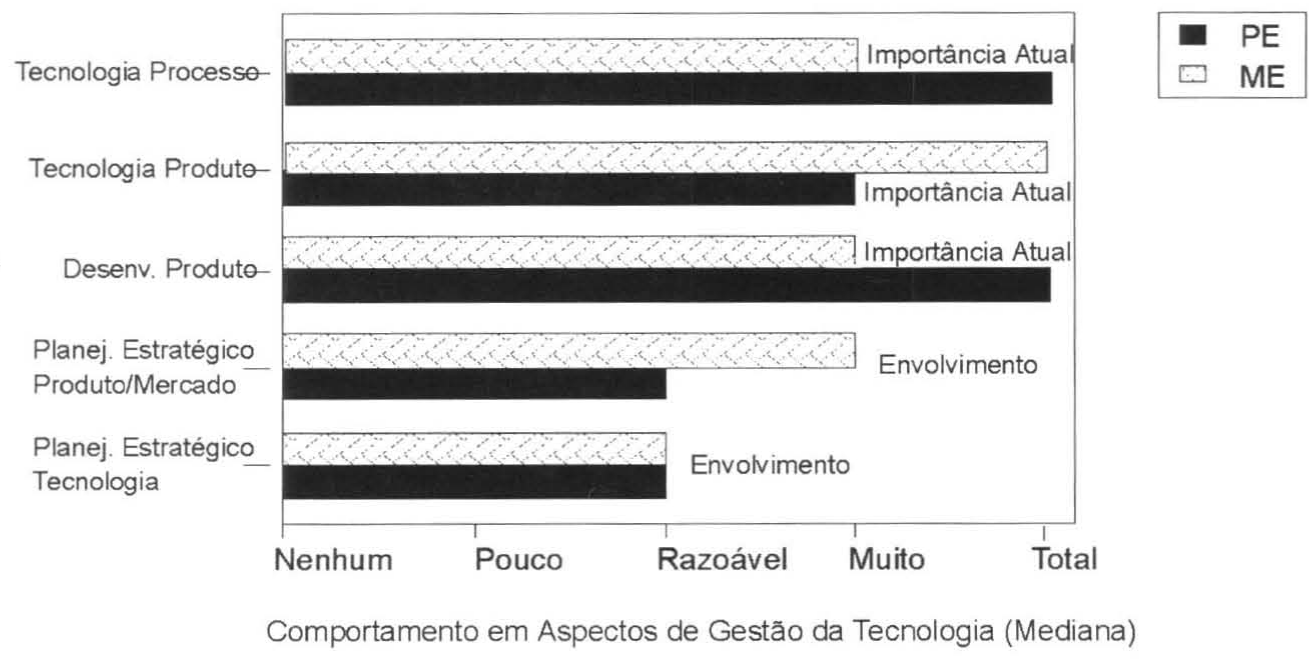

Figura 6: Comportamento das Empresas em Aspectos da Gestāo da Tecnologia

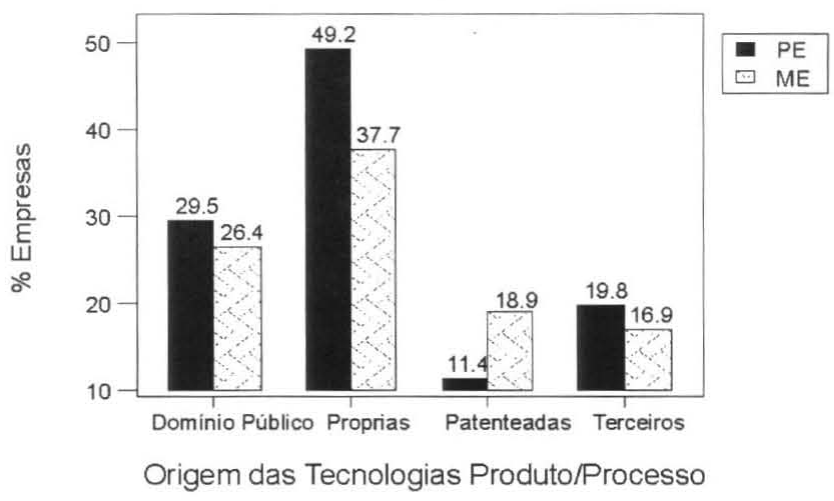

Figura 7 - Origem das Tecnologias em Pequenas e Médias Empresas 


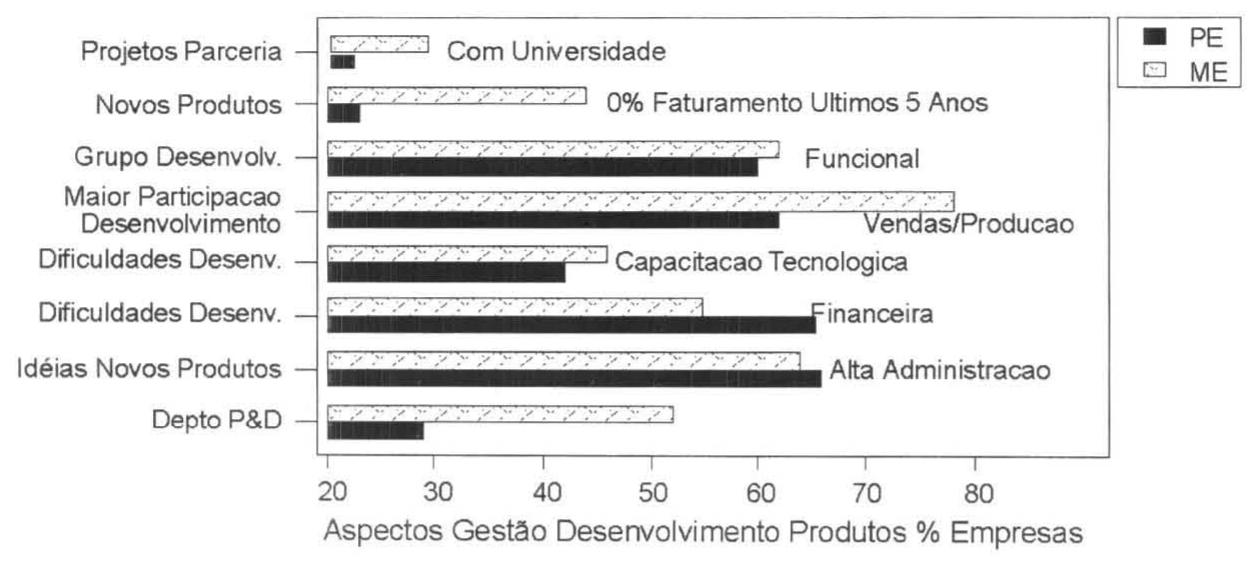

Figura 8 - Aspectos do Desenvolvimento do Produto nas Empresas

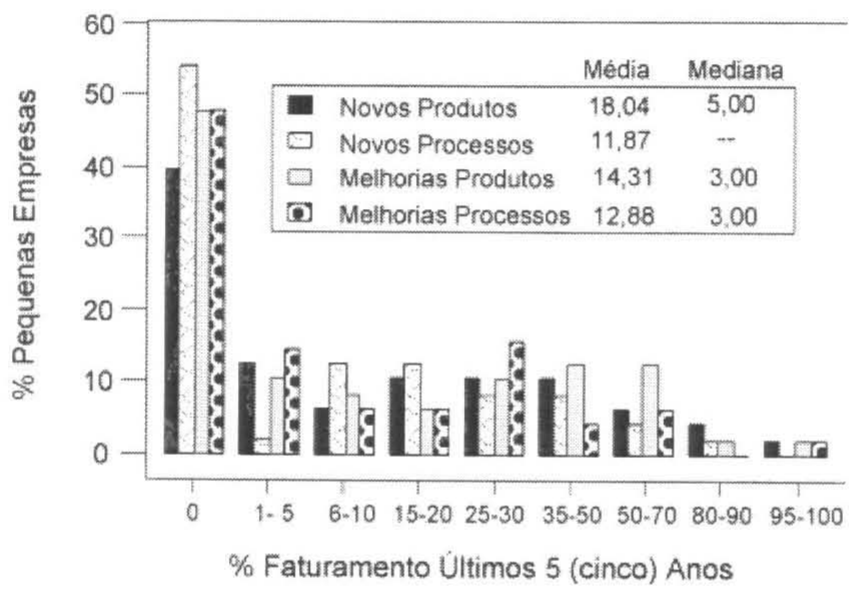

Figura 9 - Faturamento \% Últimos 5 Anos em PE

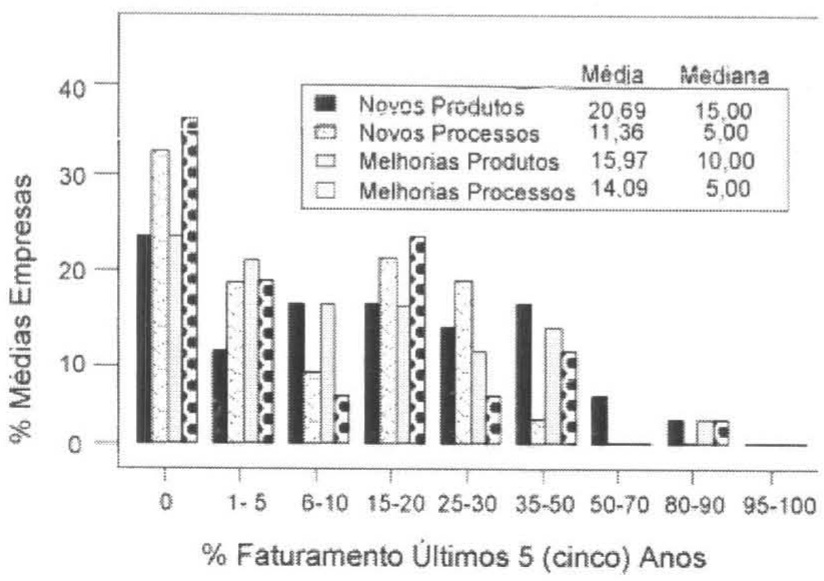

Figura 10 - Faturamento \% Últimos 5 Anos em ME 
Os gráficos anteriores por si só demonstram o estágio atual em que se encontra a maioria das organizações pesquisadas, ou seja, limitações em vários aspectos correlacionados à gestão da tecnologia, apesar da importância atual dessa área para as empresas americanas, japonesas e européias, como relatam Etzkowitz; Leydesdorff(1998) e Roberts(1995). Na Figura 6 os intervalos de confiança, relacionados à prática de planejamento estratégico de tecnologia nas empresas, estão entre pouco e regular na escala ordinal utilizada.

No campo da interação empresa-universidade, a análise do levantamento "survey" indicou os dados apresentados nas Figuras 11 e 12 onde se constata interesse das empresas em algumas áreas de formação da universidade, mas por outro lado, outros dados indicam pouca prática em projetos-parceria de pesquisa com a universidade.

As entrevistas semi-estruturadas do projeto de pesquisa qualitativa foram conduzidas em 15 empresas, após a análise do "survey", permitindo o direcionamento de questões específicas nas áreas de tecnologia $e$ desenvolvimento de produtos. No campo de "planejamento e gestão" constatou-se limitações em nível estratégico, para produto/mercado, para tecnologia, para avaliação de riscos, para acesso a sistemas de informação, e para treinamento de pessoal, além da centralização na alta administração. No campo "aspectos estruturais" constatou-se também limitações em aspectos organizacionais, em recursos computacionais, em recursos para desenvolvimento e treinamento de pessoal; preocupação com a pós-venda como um fator positivo. No campo "processo de desenvolvimento", devido às limitações no planejamento, constatou-se pouca integração entre tecnologia e aspectos de produto/ mercado, o que poderá significar projetos de altos riscos, de acordo com a concep̧̧ão de Wheelwright; Clark(1992). O desenvolvimento de novos produtos não é atividade rotineira para essas organizações e existindo muitas limitações em capacitação tecnológica e na área de marketing. Todas as empresas consideraram

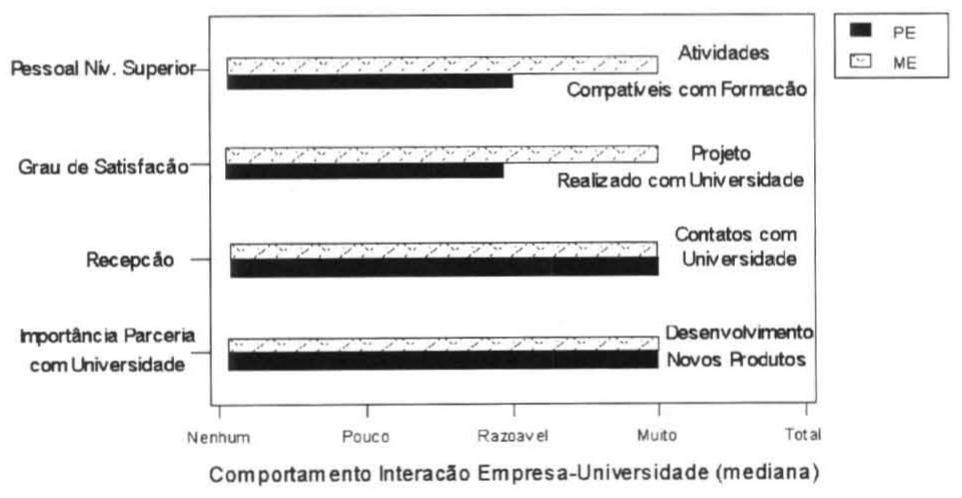

Figura 11 - Comportamento das Empresas na Interação com a Universidade

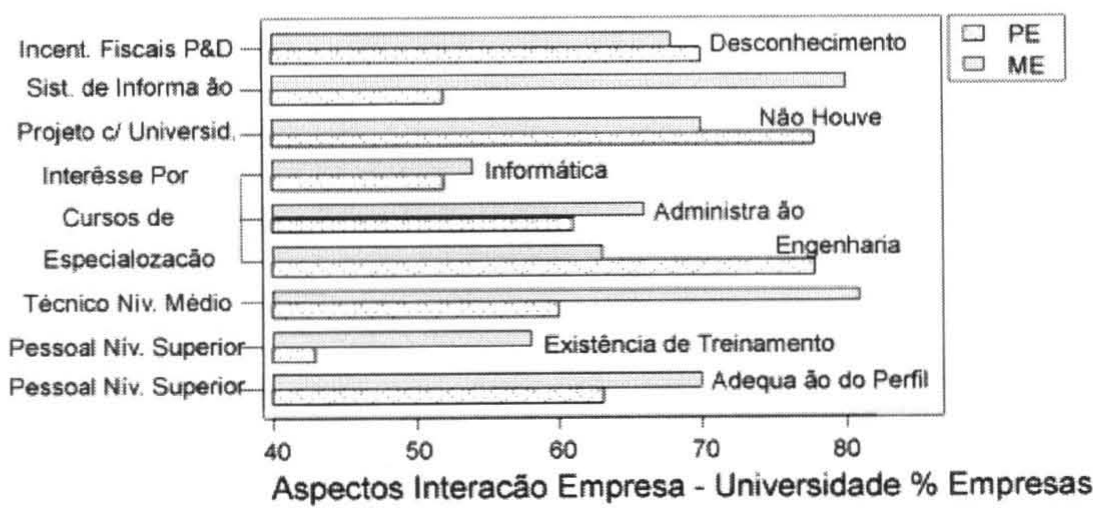

Figura 12 - Aspectos da Interação Empresa - Universidade 
importante a existência de um COD na relação interorganizacional com a universidade, no campo de desenvolvimento de novos produtos e melhorias em produtos e processos.

As entrevistas semi-estruturadas do projeto de pesquisa qualitativa, conduzidas nas universidades com 22 pesquisadores das áreas de engenharia, bem como com os órgãos de cooperação, revelaram aspectos importantes. A maioria dos projetos envolve pesquisa aplicada, embora essa característica não signifique obrigatoriamente aplicação externa à universidade. Pode-se dizer ao redor de $50 \%$ dos pesquisadores realizam pesquisas acadêmicas sem objetivos diretos de aplicação dos resultados na comunidade, visando somente publicação. Essa categoria de pesquisadores encontra dificuldades para definição de suas próximas pesquisas. A outra categoria de pesquisadores define suas pesquisas visando aplicação direta na comunidade, mas ainda dentro do modelo linear, com a maioria não conseguindo viabilizar aquela aplicação por diferentes razões. São raros os pesquisadores que geram sua pesquisa em modelo não linear e conseguem transladar os resultados para a comunidade. O planejamento da pesquisa em curto prazo é uma característica comum, exceto para pesquisadores com linhas de pesquisa definidas. A interação das atividades de graduação e pósgraduação com a industria ainda é limitada, exceto em estágios. A maioria dos pesquisadores indicou que utiliza sua experiência em pesquisa para as atividades de ensino, mas a participação de alunos de pós-graduação em suas pesquisas ainda é um aspecto controvertido. $O$ projeto de pesquisa multidisciplinar, com pesquisadores de áreas distintas, ainda não é prática comum dentro da universidade, prevalecendo a pesquisa individual. Como resultado da orientação das pesquisas, a pesquisa interativa com empresas ou outras instituições, ainda é pouco praticada. A maioria dos pesquisadores considera precária a integração entre os profissionais da universidade e da indústria.

As atividades de extensão dos pesquisadores são esporádicas, e se concentram em sua maior parte em cursos de curta duração e consultorias, com periodicidade irregular. Por outro lado, pode-se dizer que $50 \%$ dos pesquisadores indicaram ter participado ou participam de algum projeto de pesquisa para empresas, envolvendo principalmente grandes empresas. Os pesquisadores entrevistados caracterizaram suas experiências em atividades de extensão como boas, ou poucas, ou problemáticas, constituindo-se como principais motivações, a de ordem financeira e a atualização profissional.

Embora o projeto de pesquisa seja uma atividade rotineira dentro da universidade, são raras as unidades que planejam cursos de gerenciamento de projetos para os pesquisadores. A maioria dos pesquisadores entrevistados ainda não tem envolvimento com direitos de propriedade industrial. Pode-se dizer que os resultados da pesquisa, após a conclusão do projeto, são raramente avaliados visando aplicação externa, dentro do modelo de gestão existente nas universidades e nas agências de fomento. Na maioria dos casos, a publicação em periódicos é considerada, dentro da universidade, como o resultado final do projeto de pesquisa, e para as agências de fomento o projeto termina quando são aprovados os relatórios finais, técnico e financeiro. A maioria dos pesquisadores indicou que não existe interação com organismos de desenvolvimento regional. Os modelos de financiamento para projetos de pesquisa, indicados pelos pesquisadores, se concentram, em sua maior parte, na agência de fomento convencional, estadual ou federal, com reduzida participação da iniciativa privada.

A organização departamental das universidades pesquisadas é similar, direcionando-se muitas vezes para vários módulos com relativa autonomia, e distribuídos pelas sub-áreas. Cada módulo desenvolve suas linhas de pesquisa, dificultando muitas vezes o projeto de pesquisa multidisciplinar dentro do próprio departamento. Mesmo no interior desses módulos, a pesquisa individual e disciplinar prevalece.

A disponibilidade de recursos humanos e materiais, para projetos de pesquisa, é um aspecto controvertido, pois para alguns pesquisadores os maiores problemas estão nos recursos humanos, enquanto para outros em recursos materiais. Em função das dificuldades de financiamento externo à universidade, a maioria dos projetos é conduzida com limitação de recursos. Os projetos de pesquisa de maior porte somente são conduzidos com recursos financeiros externos à universidade, através de agências de fomento, organizações ou grandes empresas, e quando envolvem agrupamento de pesquisadores e técnicos, geralmente também ocorrem problemas de gerenciamento $e$ de recursos humanos. Dentro da estrutura organizacional departamental existente, os pesquisadores indicaram 
que existe flexibilidade, disponibilidade, e capacitação para desenvolvimento de projetos de pesquisa em parceria com empresas e organizações.

No que se refere à estrutura disponível de sistemas de informação, os pesquisadores consideram muito deficientes, quer seja em bancos de dados ou rede informatizada. A Internet foi referenciada com limitação de qualidade e disponibilidade de informação. Por outro lado, a maioria dos pesquisadores afirma dispor de pouca, ou nenhuma informação, sobre os projetos de pesquisa em execução em sua universidade, e que as informações das necessidades ou interesses das empresas, em seu campo de pesquisa, somente são acessíveis por esforços individuais. Praticamente todos os pesquisadores consideram o fator "comunicação" limitado, tanto entre os professores de seu departamento, como entre os departamentos, e entre a universidade e comunidade.

Todo o processo de pesquisa tem como ponto de partida a definição do que será pesquisado. A tomada de decisão, na maioria das vezes, é individualizada através de um único pesquisador, e influencia todo o processo. Essa independência constitui um fator importante para as atividades de pesquisa na universidade. Embora os riscos e incertezas sejam características inerentes à pesquisa, o grau de informação à disposição do pesquisador poderá minimizá-los, pois as questões "o que ?", "por que?", e "como?" são relevantes. Todavia, uma quarta questão importante, o "para que ?", não é muito praticada, dentro do enfoque de aplicação industrial e modelo não linear. Uma parte dos pesquisadores entrevistados encontrou dificuldades para posicionar "como" definem seus projetos de pesquisa, $e$ "o que ocorre" após e com a conclusão do projeto.

A formalização de uma proposta de projeto de pesquisa depende essencialmente do pesquisador que tomou a decisão de conduzir aquela pesquisa e das características do projeto, como recursos necessários, recursos disponíveis e fontes de financiamento. Os pesquisadores indicaram dificuldades na escolha da agência de fomento e suas linhas de financiamento, no preenchimento das propostas muitas vezes complexas, $e$ no trâmite burocrático, mesmo para os projetos

financiados com recursos "sem retorno" das agências, geralmente dentro do modelo linear. A complexidade aumenta para projetos-parceria envolvendo universidade e empresas, através de financiamento de recursos "com retorno" total ou parcial.
A indicação do comportamento dos pesquisadores, no que se refere aos procedimentos após a conclusão do projeto, se concentra principalmente no item "publicação", pois a maioria dos projetos é acadêmica. Todavia, uma parte dos pesquisadores já começa a visualizar seu projeto como atividade meio, desde sua concepção, dentro de modelo não linear, com desenvolvimento dentro da universidade ou com projetos-parceria. A análise de pré-viabilidade técnicoeconômica é raramente praticada durante a execução do projeto, por estimação dos parâmetros, ou após a conclusão do projeto, com os parâmetros já disponíveis. Evidentemente, a conclusão de um projeto de pesquisa na universidade, dentro do modelo linear, não gera um produto ou informação de valor comercial, mas um "protótipo de laboratório", no caso de manufaturados, o qual ainda deve ser viabilizado pelo desenvolvimento $e$ análises conjunturais técnico-econômicas e de mercado. Por esse motivo, a maioria das patentes geradas dentro das universidades não são utilizadas. Os pesquisadores, com experiência em projetos-parceria com empresas, indicaram a importância da comunicação interdisciplinar na universidade, pela multiplicidade das áreas envolvidas. Também assinalaram a importância da confiabilidade e sigilo na execução dos projetos.

A participação de alunos de graduação e pós-graduação em projetos-parceria, pode ser considerada reduzida, pois os pesquisadores entrevistados não conseguiram definir com precisão aquela participação.

Similarmente às entrevistas nas empresas, foram também apresentados aos pesquisadores os aspectos preliminares do modelo de gestão relacionado ao COD, havendo concordância em vários aspectos e sugestões.

As entrevistas com os órgãos de cooperação universidade - empresa das universidades não procurou delinear tendência, mas principalmente as dificuldades desses setores nas atividades de interface entre os meios acadêmico e empresarial. A vinculação à alta administração da universidade gera dependência da política predominante, função das várias correntes de pensamento ou filosofias existentes dentro do meio acadêmico. Pode-se dizer que as dificuldades desses setores envolvem duas dimensões: "interna" $e$ "externa". Na dimensão interna destaca-se a organização funcional e a limitação no gerenciamento da informação e comunicação, fatores esses relevantes para suas atividades. $O$ acesso à informação de projetos 
de pesquisa em andamento ou concluídos, dentro da universidade, não tem sido uma tarefa simples, apesar de fundamental para suas atividades. O gerenciamento da interface de projetos-parceria tem sido problemático, em função dos interesses peculiares da universidade $e$ das empresas, envolvendo dificuldades como: problemas de credibilidade mútua; não cumprimento de contratos; imediatismo das empresas; burocracia mútua; manejo das relações entre o pesquisador $e$ pessoal da indústria; negociação de direitos de propriedade industrial; despreocupação dos pesquisadores em relação à propriedade intelectual; conflitos nos direitos dos resultados do projeto; e linhas de financiamento para os projetos, entre outras. Nesse contexto, um aspecto relevante que já abordamos anteriormente, é que a maioria dos resultados das pesquisas desenvolvidas ainda está em forma de "protótipos de laboratório", sem análise conjuntural e de pré-viabilidade de desenvolvimento, etapas essas essenciais para viabilizar a negociação com as indústrias através desses órgãos. Constatamos esforços desses órgãos, nas três universidades, em agilizar suas atividades, visando melhorar os serviços prestados para os docentes, através de bancos de dados via Internet, em áreas de legislação de propriedade industrial, incentivos fiscais para a pesquisa, treinamento de pessoal para cooperação, padronização de procedimentos para projeto-parceria, ou seja, voltados em sua maior parte para o ambiente interno. Na realidade, esses órgãos ainda não conseguiram ampliar sua atuação no meio acadêmico, envolvendo também aspectos de comunicação e credibilidade. Assim, há necessidade de reavaliação do valor agregado das atividades desses órgãos dentro da interface universidade-indústria, tanto para a universidade, como para as indústrias, tendo em vista a dinâmica do meio ambiente externo atual.

$\mathrm{Na}$ dimensão externa, o gerenciamento da informação $e$ a comunicação também são fatores relevantes, principalmente dentro do modelo onde a parceria é acionada pelas empresas. A disponibilidade de sistemas de informações seletivas é limitada, recaindo quase sempre na Internet, apesar da indicação de que a metade das médias empresas e a maioria das pequenas empresas ainda não esteja conectada. Ademais, o perfil do profissional para gerenciar a interface universidadeempresa, apesar de sua importância, ainda é problemático, tanto do lado da universidade, como das empresas. A prospeç̧ão externa de oportunidades de parceria está diretamente interligada à prospecção interna de disponibilidades e competências, e somente poderá ser bem sucedida através de pessoal com perfil profissional adequado. Esse perfil deve ser uma composição de empreendedor, pesquisador e de relações industriais e institucionais, dificilmente encontrado na universidade.

As principais categorias de cooperação, ordenadas quantitativamente, envolvem : consultorias, informações técnicas, cursos de curta e média duração, laudos técnicos, projetos-parceria de pesquisa, análise de invenções. O projeto-parceria ainda é pouco praticado dentro das universidades consultadas, e de acordo com um dos coordenadores entrevistados, menos de $1 \%$ dos pesquisadores tem participação nesses projetos, embora haja interesse.

Os projetos-parceria de pesquisa geralmente são acionados pelas empresas, ou por indicação do pesquisador, envolvendo em sua maior parte as médias e grandes empresas, com a utilização de recursos oriundos em parte de incentivo fiscal, fundo perdido, ou com juros reduzidos. A pesquisa, em sua maioria, é conduzida e coordenada pela universidade, até o chamado "protótipo de laboratório" ou similar. Os entrevistados indicaram que a eficácia (tempol qualidade) na execução desses projetos pode ser considerada média. Os direitos de propriedade industrial, resultantes da pesquisa, seguem a legislação vigente nesse campo e alguns aspectos peculiares de cada universidade, pois envolve a instituição e o pesquisador, podendo também envolver a empresa quando de projetos - parceria. Quando os contratos entre as partes não abordam claramente esse aspecto, podem ocorrer conflitos de interesses.

Ao serem indagados se tinham conhecimento, nos últimos 2(dois) anos, de algum produto desenvolvido por parceria empresa-universidade, que tenha resultado em sucesso comercial, os entrevistados informaram desconhecer ou não dispor de dados. Por outro lado, em um período maior, informaram que alguns projetos de pesquisa resultaram em desenvolvimento de novos produtos, tanto no modelo linear como não-linear.

Por último, similarmente ao que acorreu com as entrevistas com pesquisadores e empresas, foram apresentados os aspectos preliminares do modelo de gestão relacionado ao COD. Os entrevistados 
consideram adequado ou razoável o modelo, e procederam algumas observações : divulgação do modelo para associações e órgãos como CIESP, $S E B R A E$, etc, visando apoio para implantação; qualquer modelo, nesse campo, somente se viabilizará conquistando a credibilidade da comunidade interna da universidade; o modelo deve adaptar-se a aspectos regionais.

\section{Conclusão}

$O$ presente trabalho analisou a interface empresauniversidade e a gestão da tecnologia em pequenas e médias empresas brasileiras tradicionais do setor de manufaturados. Existem limitações dessas empresas no campo da gestão da tecnologia, quer seja para as tecnologias existentes de produtos/processos, mas principalmente para novas tecnologias, denotando dificuldades nesse importante campo dentro do contexto atual da economia globalizada. $O$ desenvolvimento de novos produtos/processos com novas tecnologias, $e$ mesmo com tecnologias existentes, não é prática rotineira dentro dessas organizações, por diferentes aspectos. Entre eles a limitação em capacitação tecnológica, em estrutura, em planejamento e gestão, em informação tecnológica e de mercado, e em disponibilidade financeira. A adoção da moderna gestão da qualidade, no contexto das tecnologias de produto/ processo existentes na empresa em um determinado instante, poderá aumentar a competitividade dessas empresas em curto prazo, porém não será suficiente a médio e longo prazo, onde o enfoque em aspectos tecnológicos é fundamental. Uma empresa que investe somente em importação de equipamentos, como solução de seus problemas, poderá "estar" competitiva, mas iniciando um processo de dependência, com sérias repercussões futuras, caso não detenha as tecnologias de processo. As tecnologias de produto estão expostas no mercado, enquanto que as tecnologias de processo são internas à empresa. A competitividade de uma empresa, entre outros aspectos, está no domínio das tecnologias de produto/processo, e no conteúdo tecnológico presente. Esse conteúdo tecnológico, pelo menos por algum tempo, poderá ser o diferencial da empresa com os concorrentes. Assim, quando uma empresa desenvolve uma tecnologia, reduz sua vulnerabilidade, e poderá ser competitiva por tempo maior, quando em comparação com aquisição ou licenciamento de tecnologias. No mercado globalizado a transferência de tecnologia entre organizações é limitada, pois teoricamente todas as empresas podem atuar em todos os mercados, e quem dispõe de um conteúdo tecnológico importante irá explora-lo através de produtos/processos e não transferilo para outras organizações, embora essa transferência, quando ocorra, seja sempre parcial se analisarmos mais profundamente o conceito da palavra tecnologia. $\mathrm{Na}$ realidade, uma transferência de tecnologia só ocorre quando o produto é uma "commodity", ainda assim parcialmente, significando competitividade aparente $e$ transitória.

Um caminho ainda pouco utilizado pelas empresas tradicionais, na formação de capacitação tecnológica, é a parceria com universidades e institutos, dentro de certos critérios, pois a pesquisa aplicada é somente o início do processo de desenvolvimento, cuja prática não é comum dentro das universidades. Esse processo de desenvolvimento, atualmente agilizado através de métodos e ferramentas modernas, poderá gerar com sucesso produtos/processos na saída do sistema, desde que em sua entrada haja disponibilidade de conteúdo tecnológico, com interesse mercadológico e participação direta de empresas. Esse conteúdo tecnológico poderá ser desenvolvido com colaboração da universidade, mas o "start-up" deve ser dado pela empresa, pois somente ela pode ter visualização de empreendimento de mercado e de potencial de lucro. Assim, a participação da universidade está limitada à pesquisa básica ou aplicada, ou seja o início do processo de desenvolvimento, dentro do modelo não linear em parceria com empresas. Com a disponibilidade preliminar dos parâmetros da tecnologia oriunda da pesquisa, a próxima etapa é o desenvolvimento de produto/processo, onde os investimentos são mais elevados, porém resultando no final do processo a saída do produto/processo para satisfazer as necessidades da comunidade. Nas condições atuais, o moderno processo de desenvolvimento é também não linear, multidisciplinar, exigindo uma infraestrutura raramente encontrada nas organizações, com maior limitação para pequenas e médias empresas. $E_{\text {exatamente nessa fase }}$ do processo de desenvolvimento em que reside o ponto "chave" para viabilização de tecnologias/produtos/ processos dentro do contexto atual da economia globalizada. É interessante ressaltar que as agencias de fomento tradicionais ainda não perceberam esse fato, pois não financiam essa etapa. Como esses órgãos somente financiam o início do processo, ou seja, as 
pesquisas, certamente estão operando no modelo linear, considerando como fim o "final da pesquisa", e não considerando pesquisa como "atividade meio" visando o uso do conhecimento pela comunidade. Em outras palavras, "o resultado de uma pesquisa é informação, $e$ essa informação não está se transformando em conhecimento, pois não é usada pela comunidade".

Dentro desse contexto, o "modelo central" sugerido para esse sistema se concentra na relação interorganizacional empresa-universidade, o qual designamos de CODCentro Operacional de Desenvolvimento, cuja função principal é executar e gerenciar o processo de desenvolvimento de novos produtos/processos em parceria com as empresas, principalmente de pequeno $e$ médio porte, cujo detalhamento é apresentado em Silva(1999). Quando da não disponibilidade de tecnologia, poderão ser gerados projetos-parceria de pesquisa empresa-universidade, cujos resultados serão aplicados no processo de desenvolvimento CODEmpresa. O COD não é um centro de pesquisa cooperativa, pois não desenvolve ou executa pesquisa tecnológica, mas um centro de desenvolvimento que inicia o seu processo a partir da já disponibilidade de tecnologias, novas ou existentes, visando incorpora-las em um novo produto ou novo processo de valor comercial. Essa constitui a principal diferença, $e$ fundamental, entre o COD e os centros de pesquisas cooperativos IUCRCs ou ERCs dentro das universidades públicas americanas.

Outros aspectos importantes da gestão da tecnologia na empresa também poderão contar com o apoio do COD, como o treinamento de gerentes para desenvolvimento, a prospeç̧ão e informação tecnológica, além da intermediação de consultoria da universidade em diferentes áreas. Em nível de universidade agrega-se a prospecção $e$ análise dos resultados de pesquisas concluídas pelo modelo linear, a divulgação interna $e$ externa ao Campus, e o treinamento de pesquisadores em gerenciamento de projetos, além de outras atividades correlacionadas às oportunidades de desenvolvimento de projetos-parceria de pesquisa com empresas. Em nível de empresa a difusão da prática moderna de desenvolvimento, treinamento de gerentes de desenvolvimento, e intercâmbio de informações em ciência e tecnologia.

Essa interface poderá incentivar o modelo de pesquisa não linear nas universidades e colaborar com o desenvolvimento regional, tendo como resultado final a geração de capacitação tecnológica e competitividade das empresas, aspectos estes importantes na economia globalizada, não só para o setor de manufaturados como também para outros setores, como por exemplo em processos contínuos $e$ bens de capitais. Acreditamos que as agencias de fomento à tecnologia precisam fazer uma auto-avaliação, e reformular suas políticas para um mundo globalizado onde a linearidade não mais existe no campo da tecnologia.

\section{Bibliografia}

BOWERS, L. J. University-industry technology transfer: basics principles. SRA Journal, v. 23, 1991, p. 53-56.

BRYMAN, A. Research methods and organization studies. Londres, Unwin Hyman, 1989.

DURHAM, E.R. Avaliação e relações com o setor produtivo. Revista Educação Brasileira, v. 12, n.24, 1990, p. 37-64.

ETZKOWITZ, H. ; LEYDESDORFF, L. "A triple helix of university-industry-government relations: introduction". Industry \& Higher Education, v. 12, n. 4, 1998, p.197258.

GAZZINELLI, P. A tecnologia como fator de competitividade nas empresas. ABM News, Associação Brasileira de Metalurgia e Materiais, ago. 1999, São Paulo.

GEISLER, E. Organizational and managerial dimensions of industry-university-government $R \& D$ cooperation : a global perspective. Cleveland-Ohio, Case Western Reserve University, mar. 1995.

GRIGG, T. Adopting an entrepreneurial approach in universities. Journal of Engineering and Technology Management, v. 11, n. 3 e 4, 1994, p. 273-98.

HAYES, B. E. Measuring customer satisfaction : development and use of questionnaires. Wisconsin-USA, ASQC Quality Press, 165p., 1992.

KRUGLIANSKAS, I. Tornando a pequena e média empresa competitiva. São Paulo, Instituto de Estudos Gerenciais e Editora, 1996.

LINK, N. Transferência de Tecnologia. Seminário na Escola Politécnica, Departamento de Engenharia de Produção USP, São Paulo, jul. 97.

MENEGHEL, S. M. ; GOMES, E. J. Relações da Funcamp com o meio externo no período 1982-1995. In: Simpósio de Gestão da Inovação Tecnológica-USP, 19. , São Paulo, 1996. Anais, v.1, p.598-610. 
MERLI, G. Comakership: a nova estratégia para o suprimento. Gregório Bouer. Rio de Janeiro,, Qualitymark Ed., 1994.

MORAES, R.; STAL, E. Interação empresa-universidade no Brasil. Revista de Administração de Empresas-RAE, v.34, n.4, jul./ago. 1994, p. 98-112, São Paulo.

PLONSKI, G. A. Cooperação empresa-universidade na ÍberoAmérica : estágio atual e perspectivas. Revista de Administração, v.30, n.2, abr./jun. 1995, p. 65-74,.

PORTER, M. E. What is strategy? Harvard Business Review . v. 96, n.6, 1996, p. 61-78.

ROBERTS, E.B. Benchmarking the strategic management of technology-II. Research Technology Management, v.38, n.2, march./april 1995, p. 18-26.

SCHUETZE, H. G. Innovation systems, regional development, and the role of universities in industrial innovation. Industry and Higher Education, v. 10, n. 2, apr. 1996, p. $71-8$.

SIEGEL, S. Estatística não-paramétrica. São Paulo, Ed. McGraw-Hill, 1975.

SILVA, J. C. T. Interface universidade-empresa. III Simpósio de Engenharia de Produção, Unesp, Bauru-SP, Anais em CD Rom, nov. 1996.

SILVA, J. C. T. Modelo interativo empresa-universidade no desenvolvimento de produtos. São Paulo, 1999. 163p. Tese (Doutorado) - Departamento de Engenharia de Produção, Escola Politécnica, Universidade de São Paulo.

SILVA, J. C.T.; PLONSKI, G.A. Gestão da tecnologia : desafios para pequenas e médias empresas, Revista Produção, ABEPRO, v.9, n.1, out. 99, p. 23-30, Rio de Janeiro RJ.

STREHARSKY, C. I. Creating ethical university-industry partnerships. SRA Journal, v. 25, n.1, 1993, p. 23-30.

WHEELWRIGHT, S. C.; Clark, K. B. Revolutionizing product development. New York, The Free Press, 1992.

ZAGOTTIS, D.L. A interação entre universidade e o sistema produtivo. Trabalho apresentado a Convencion Panamericana de Ingenieros-UPADI, 24. , San José da Costa Rica, 1996.

ZIMAN, J. A neural net model of innovation. Science and Public Policy. View Point, v.18, n.1, 1991, p. 65-75. 